\section{AOA takes issue with AMA "paranurse" proposal}

The American Osteopathic Association House of Delegates, meeting in Miami Beach in August 1988, unanimously passed a resolution opposing the American Medical Association's recent proposal calling for the creation of a new type lesser trained paranurse, called a registered care technologist (RCT). This person would assume many bedside nursing functions.

The AOA resolution stated that the creation of this new category of health care worker would exacerbate the shortage of registered nurses by delay and misallocation of resources. Furthermore, it would impose an inappropriate, potentially confounding additional layer in the provision of health care services. This, in turn, would erode the quality of inpatient care. The resolution called for appropriate changes in nursing compensation, professional incentives, and augmented public and private funding for nursing education to address the principal root causes of the growing registered nurses shortage.

According to Joseph W. Stella, DO, outgoing AOA president and author of the resolution, the current shortage is comparable to the physician shortage in the $1960 \mathrm{~s}$. He explained, "the solution to the physician shortage was not to produce second class physicians to fill the gap, but to increase the number of first class physicians. Instead of looking to shortcut any kind of care for our patients, we should look to the same type of solutions that were implemented in the late $60 \mathrm{~s}$ and early $70 \mathrm{~s}$. Federal funding was used to create more slots in medical schools, which I have not seen proposed for nursing schools."

The American Osteopathic Association is committed to the highest quality of health care for the American people. We firmly believe that the registered nurse, as the physician's essential professional partner, plays an intrinsic role in assuring that quality care.

Leaders of the American Nurses' Association (ANA) have responded positively to the AOA proposal and expressed appreciation for our support. The ANA has been studying the RCT proposal and its impact on the nursing profession. On Sept 27, ANA officials will meet with AOA leaders and members of several health care groups to develop a formal response to AMA's proposal.

THOMAS WESLEY ALLEN, DO, FACOI

\section{Mission accomplished?}

Too many persons perceive osteopathic medicine as "regular" medicine plus structural diagnosis and manipulative treatment (OMT). Unfortunately, it is often practiced in that context. Therefore, we need an occasional reminder that structural diagnosis and OMT were but one way of demonstrating Andrew Taylor Still's basic philosophy.

From the very beginning, the objective of the first osteopathic medical school was to "improve [the] present system of surgery, obstetrics, and treatment of diseases and generally place the same on a more rational and scientific basis...." Since its inception, then, osteopathic medicine was considered a system embracing the arts and sciences in medical practice. It was never a "nonmedical" school but rather a philosophy for all of medicine committed to improve its practice. It was a statement of responsibility and challenge.

Much has changed since then. Through the years there has been a tendency to emphasize the structural and OMT phases of our profession as the difference between allopathic and osteopathic medicine. While this is a significant contribution to our profession, focusing on musculoskeletal structure and OMT in diagnosis and treatment can lead us to complacency, believing our mission complete.

Although untrue, it's easy to understand how that conclusion is reached. Never in the history of modern medicine has so much interest been shown in OMT with all its various forms, such as muscle energy techniques, strain and counterstrain procedures, cranial, myofascial and the like. Large numbers of postdoctoral courses and instructional seminars are being held in which DOs, MDs, and other professionals are taking part. These courses are referred to as 'manual medicine' or 'musculoskeletal medicine.'

This expanded interest in OMT as a separate entity from what generally is understood as medical 